\title{
Green synthesis of silver nanoparticles by aqueous extract of Dovyalis abyssinica fruit for antibacterial applications
}

\author{
Mekuriaw Assefa Kebede ${ }^{1, *}$, Baye Sitotaw $^{2}$, Kidanemariam Teklay Hilawea ${ }^{1}$ \\ ${ }^{1}$ Department of Chemistry, Bahir Dar University, Bahir Dar, Ethiopia \\ ${ }^{2}$ Department of Biology, Bahir Dar University, Bahir Dar, Ethiopia
}

\begin{abstract}
Green biosynthesis technique was employed to synthesize silver nanoparticles (AgNPs). Fresh citrus fruit of Dovyalis abyssinica (vernacular name Koshim) tree was extracted by distilled water to obtain phenolic natural compounds that have reducing capacity of metallic ions to the corresponding metallic nanoparticles, in this case, silver ion to silver nanoparticles. The appearance of the UV-Vis absorbance peak at $430 \mathrm{~nm}$ and the color change from pale yellow reaction mixture to reddish brown colored product solution have confirmed the formation of AgNPs. FTIR data has also shown the presence of organic components from the plant with the particles that may be used as capping agents to stabilize the formed particles and to control the size. The prepared nanoparticles and the plant extract have shown antibacterial property against $E$. coli and $S$. aureus, though the effect of the AgNPs was better than the plant extract. This study contributes for the development of environmentally friendly procedures in the preparation of nanoparticles for medicine, energy or environment.
\end{abstract}

Keywords: Dovyalis abyssinica; Green biosynthesis; Silver nanoparticles; Antimicrobial activity

DOI: https://dx.doi.org/10.4314/ejst.v14i3.4

\section{INTRODUCTION}

Nowadays metallic nanoparticles have a broad application in environment, energy, and medicine (Ariga et al., 2011). Their prominent efficiency is because of improved properties on their strength, optical property, and high surface to volume ratio compared to the bulk material. Even though their synthesis involves chemical, mechanical, electrochemical or optical processes, the whole process should be efficient, easy, cost-effective and ecofriendly. Hence the development of green synthesis methods is one of the current research areas

\footnotetext{
${ }^{*}$ Corresponding author: meku.assefa65@gmail.com

(C) This is an Open Access article distributed under the terms of the Creative Commons Attribution License (http://creativecommons.org/licenses/CC BY4.0)
} 
towards the preparation of nanoparticles to minimize the effect of hazardous synthetic chemicals during the synthesis procedures.

The green biosynthesis process usually involves less toxic solvent, less reducing and capping agents to get the desired nanoparticle. Use of bio-based starting materials from plants, fungi, and bacteria as reducing or capping agents in nanomaterial synthesis is considered as a green process (Nadagouda and Varma, 2008; Kumar et al., 2013; Aramwit et al., 2014; Chowdhury et al., 2014; Mohamed et al., 2016; Siddiqi and Husen, 2016; Markus et al., 2017; Choi et al., 2018). During metallic nanoparticle synthesis, metal ions are reduced to metallic particles because of the functional groups of secondary or primary metabolites such as proteins, amines, amino acids, phenols, sugars, ketones, aldehydes, and carboxylic acids present in the plant extract. The shape and size of the synthesized nanoparticles can be managed by monitoring the temperature, $\mathrm{pH}$, reaction time, and concentration of the plant extract and metal precursor (Kaviya et al., 2011; Siddiqi and Husen, 2016).

Currently, different research reports are available on the biogenic processes for the synthesis of silver nanoparticles for different applications, mainly as potent antimicrobial agents (Songping and Shuyuan, 2005; Nadagouda and Varma, 2008; Kaviya et al., 2011; Masurkar et al., 2011; Rashid et al., 2013; RodríguezLeón et al., 2013; Aramwit et al., 2014; Chung et al., 2016; Baláž et al., 2017; Choi et al., 2018).

Biosynthesis of silver nanoparticles (AgNPs) was achieved by a novel, simple green chemistry procedure using citrus sinensis peel extract as a reducing and a capping agent (Kaviya et al., 2011). Origanum vulgare L. plant extract was also used as a reducing agent for the formation of AgNPs within 15 min with two average sizes of $38 \pm 10 \mathrm{~nm}$ and $7 \pm 3 \mathrm{~nm}$ (Baláž et al., 2017). Also, leaf extract of Ocimum sanctum was used as a reducing agent for the synthesis of platinum nanoparticles $(4 \mathrm{~nm})$ from an aqueous chloroplatinic acid $\left(\mathrm{H}_{2} \mathrm{PtCl}_{6} \cdot 6 \mathrm{H}_{2} \mathrm{O}\right)$ precursor. It was confirmed by Fourier-Transform Infrared Spectroscopy that natural compounds such as ascorbic acid, gallic acid, terpenoids, polyphenols, certain proteins, and amino acids acted as reducing agents to convert the metal ion into metallic particles (Nadagouda and Varma, 2008; Soundarrajan et al., 2012; Kumar et al., 2013).

Dovyalis abyssinica (D. abyssinica) is one of the medicinal plants found in Ethiopia. It can grow to a height of 6 to $10 \mathrm{~m}$ tree that gives yellow and edible but sour tasting citrus fruit (Kipngeno et al., 2014; Chirchir et al., 2018). However, research on the chemical composition and biological activity of this plant is limited. Some phytochemical investigation was carried using methanolic 
extract of the fruit to determine its antimicrobial activity against Staphylococcus bacteria and Trichophyton rubrum fungus (Kipngeno et al., 2014). The result revealed that the plant was a good source of natural compounds that have potent antimicrobial and antifungal effects. The phytochemical screening result has also shown the presence of alkaloids, flavonoids, tannins, phenols, saponins, and proteins (Kipngeno et al., 2014). However, there is no report on the application of D. abyssinica extracts to be used as reducing agent to synthesize metallic nanoparticles. Hence, in the current research, an aqueous extract of $D$. abyssinica fruit was used as a reducing agent to prepare silver nanoparticles. Antibacterial activity of the assynthesized AgNPs was then compared with the antibacterial activity of the crude extract.

\section{MATERIALS AND METHODS}

Silver acetate from BDH Laboratory Supplies, England; Mueller Hinton Agar from Microxpress, India; and Gentamicin from Himedia, India was used. The test strains were kindly provided by Amhara Regional State Public Health Institute, Bahir Dar, Ethiopia. Deionized water was used in all processes of the study. Fourier transmission spectrometer (FTIR) and UV-Vis spectrometers were used for analysis.

\section{Extraction procedure}

The mature fruit were collected from the tree in Bahir Dar, Ethiopia. The fruit were preserved in refrigerator at $4{ }^{\circ} \mathrm{C}$. Decoction technique (Shija et al., 2020) was employed to isolate water soluble compounds from the plant. Typically, the cleaned and peeled fruit were crushed by using an electric blender to get the crude juice. Then, $50 \mathrm{~g}$ of juice was diluted with $200 \mathrm{~mL}$ distilled water in $1 \mathrm{~L}$ Erlenmeyer flask and heated by a water bath at $60{ }^{\circ} \mathrm{C}$ for $1 \mathrm{~h}$. The hot solution was filtered to remove the residue through microfiltration setup and obtain crude extract filtrate which was kept in the refrigerator for the next process.

\section{Reduction procedure}

The reduction process was performed according to Baláž et.al. (2017) procedure with little modification. Hence, $0.3 \mathrm{~g}$ of silver acetate was dissolved in $100 \mathrm{~mL}$ distilled water to prepare the metal precursor solution from which $45 \mathrm{~mL}$ was taken and heated in the water bath at $80^{\circ} \mathrm{C}$ for $10 \mathrm{~min}$ with occasional shaking. Then, $5 \mathrm{~mL}$ of the fruit extract was added slowly and the mixture was further heated for $15 \mathrm{~min}$ to finish the reduction process. 


\section{Characterization}

UV-Vis spectrometer was used to measure absorbance and FTIR spectrometer to see the functional groups. Agar diffusion method was employed to check the antibacterial activities of both filtered crude extract and the AgNPs colloid.

\section{Antibacterial test}

The disc diffusion technique was employed to evaluate the antibacterial activity of the crude aqueous extract and AgNPs against Escherichia coli (E. coli) and Staphylococcus aureus (S. aureus). A $24 \mathrm{~h}$ old bacterial culture of both strains was spread on Mueller Hinton agar using a sterilized cotton swab. Seven $\mathrm{mm}$ discs were prepared from Whatman filter paper and sterilized by autoclave at $121{ }^{\circ} \mathrm{C}$ for $15 \mathrm{~min}$. The discs were then immersed into the test solutions and aseptically put on the agar plates that contain the bacteria. Gentamicin (Himedia, India) was used as positive and deionized water as negative controls. Subsequently, the plates were incubated overnight at $37{ }^{\circ} \mathrm{C}$ to observe the inhibition zone. The diameter of the clear zone was measured in millimeters using a standard ruler in triplicates.

\section{RESULTS AND DISCUSSION}

According to UV-Vis data in Figure 1, the absorbance peak at $430 \mathrm{~nm}$ is attributed to silver nanoparticles (Baláž et al., 2017). Moreover, the color change from pale yellow of the mixture of reactants to a reddish-brown colloid solution was attributed to the formation of silver nanoparticles (Inset of Figure 1). Whereas the pure plant extract exhibits two major peaks (Figure 1A) at ultraviolet regions of the measurement that are attributed to different functionalities of natural products in the extract. After the reaction, these peaks disappeared, indicating their role in the reduction of silver ions to silver metal particles.

FTIR spectra of the extract and AgNPs were recorded as shown in Figure 2. The spectrum from the extract (curve A of the figure) contains many peaks since it is a crude aqueous extract that is expected to contain many organic compounds belonging to polyphenols, alkaloids, tannins, and others (Kipngeno et al., 2014; Chirchir et al., 2018). The result shows intense absorption peak at $3427 \mathrm{~cm}^{-1}$ indicating the presence of $-\mathrm{OH}$ like polyphenols. The peak at $1724 \mathrm{~cm}^{-1}$ is typical for carbonyl groups. These two peaks together indicate the presence of carboxylic acid functionality of ascorbic acid (Songping and Shuyuan, 2005), usually found in citrus fruit such as D. abyssinica. Other peaks at 1630, 1387, 
1235 , and $1905 \mathrm{~cm}^{-1}$ revealed the diversity of functionalities in the extract of the fruit. However, while almost all of the functional groups in the crude extract are retained at their characteristic wave length after the reduction reaction step (curve B of the figure), the observed decrease in their absorption intensity may be taken as an indicator for their participation in the reduction reaction step of the metal ions to metal atoms.

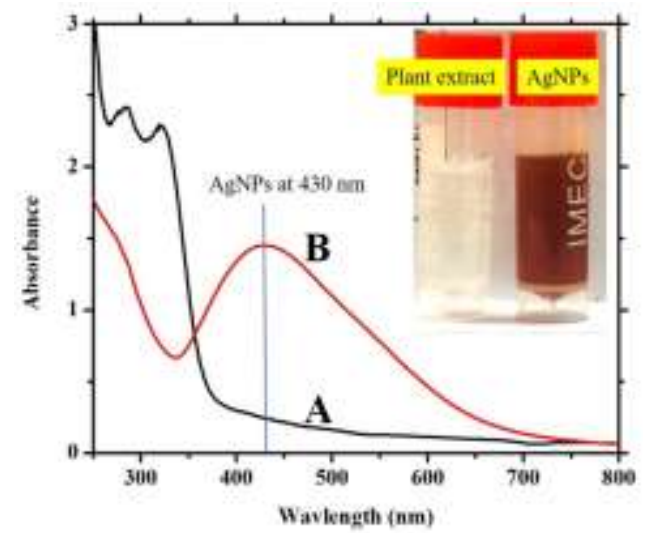

Figure 1. UV-Vis spectra of A) aqueous extract from D. abyssinica fruits, and B) as obtained AgNPs colloid solution.

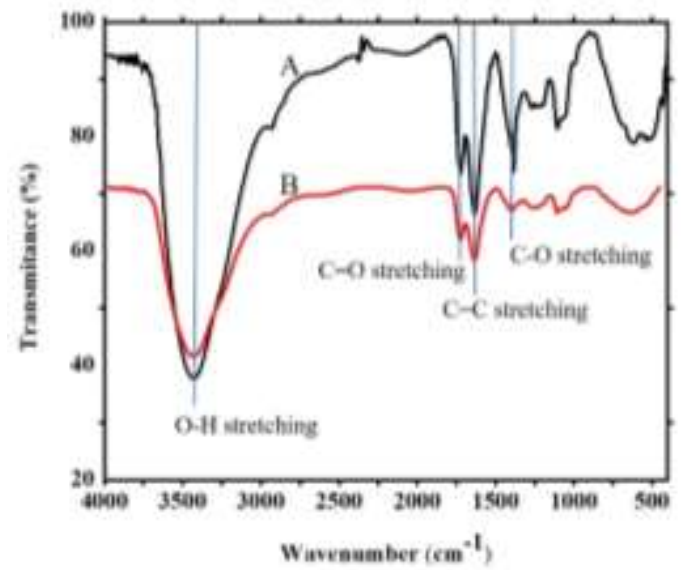

Figure 2. FTIR spectra of A) crude extract from D. abyssinica fruits, and B) AgNPs.

The presence of phenolic compounds which are known as natural reducing agents (Karsheva et al., 2013; Kumar et al., 2013; Safdar et al., 2017) in the fruit may have played a great role in the conversion of the silver ion into 
metallic silver. Moreover, the data revealed that compounds in water extract of the plant also served as a capping agent to get AgNPs.

Figure 3 shows the photo of antibacterial test result. Both the plant extract and AgNPs show potent activities against $E$. coli and $S$. aureus. As can be seen from the image and the summary result (Table 1), the extract inhibition was greater in both strains than that of AgNPs which might be due to the difference in diffusion ability of organic molecules than metallic particles. With better diffusion and contact with the active agent and the bacteria, inhibition will be better. The organic molecules could diffuse better and cover larger areas than AgNPs. However, other reports show that the inhibition performance of silver nanoparticles was comparable as to the antibacterial activity of biosynthesized silver nanoparticles against these bacteria strains (Kaviya et al., 2011). Additionally, some bacteria strain was observed within the circle of inhibition zone of the plant extract while that of AgNPs was clearer inferring the better killing ability of the particles than the plant extract. The known antibacterial drug gentamicin, which was used for comparison, effectively killed the pathogens in the samples.
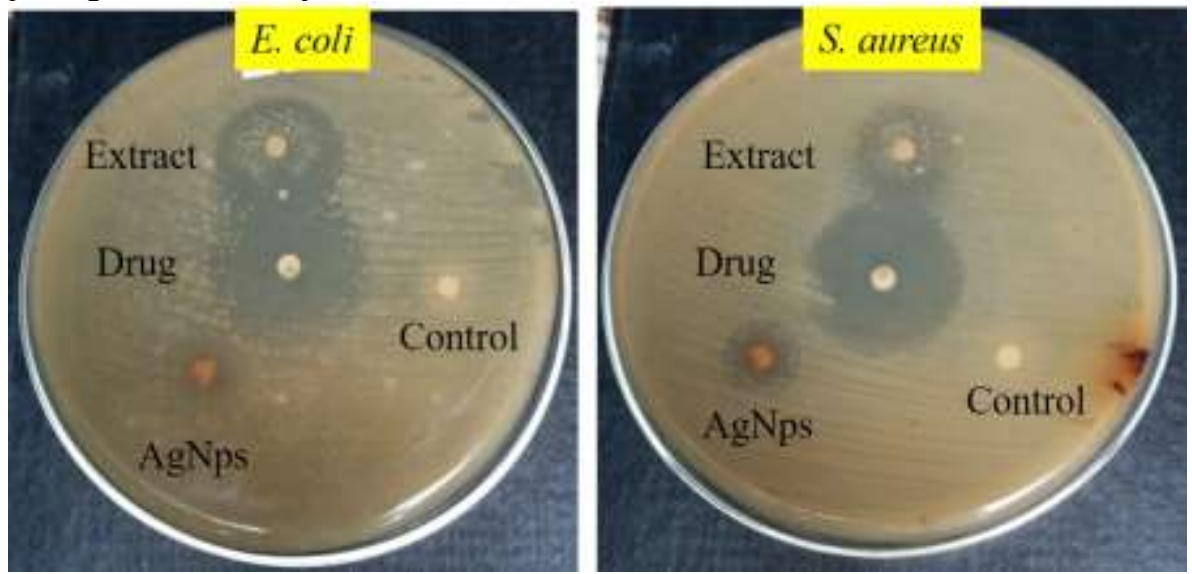

Figure 3. Image of antimicrobial activity test of A) aqueous crude fruit extract of $D$. abyssinica, and B) biosynthesized AgNPs.

Table 1. Inhibition zone summary of antibacterial activity test (in $\mathrm{mm}, \mathrm{n}=3$ ).

\begin{tabular}{lccc}
\hline Strains & $\begin{array}{c}\text { Crude } \\
\text { extract }\end{array}$ & $\begin{array}{c}\text { AgNPs } \\
\text { solution }\end{array}$ & Gentamicin \\
\hline E. coli & $29.0 \pm 0.10$ & $19.3 \pm 0.21$ & $31.6 \pm 0.06$ \\
S. aureus & $30.7 \pm 0.06$ & $23.3 \pm 0.23$ & $40.0 \pm 0.00$ \\
\hline
\end{tabular}




\section{CONCLUSION}

In this study, we confirmed that the plasmonic absorption band observed at 430 $\mathrm{nm}$ revealed the success of conversion of silver ions to silver nanoparticle colloid. The FTIR spectrum of the extract and synthesized particles has shown natural compounds with acidic functionality were observed in the aqueous extract and also retained in AgNPs. This indicates some of natural compounds may be surrounded by the particles to control the size and act as stabilizing agents. Finally, both the fruit extract and the prepared AgNPs have shown good antibacterial activity against $E$. coli and $S$. aureus. The extract had a clear zone of inhibition of $29.0 \pm 0.10 \mathrm{~mm}$ against $E$. coli and $30.7 \pm 0.6 \mathrm{~mm}$ against $S$. aureus. The AgNPs could kill bacteria with inhibition zone of $19.3 \pm 0.21 \mathrm{~mm}$ against $E$. coli and $23.3 \pm 0.23 \mathrm{~mm}$ against $S$. aureus. Therefore, the novelty of this study was the application of natural organic compounds obtained from aqueous extraction of fruit of $D$. abyssinica as reducing agents and stabilizers to synthesize silver nanoparticles that have good antibacterial effects.

\section{ACKNOWLEDGMENTS}

The authors would like to thank Tessera Alemeneh $(\mathrm{PhD})$, Material Sciences and Engineering Department, Bahir Dar University, Ethiopia.

\section{FUNDING}

No specific grant was received for this research from funding agencies in the public, commercial, or not-for-profit sectors.

\section{REFERENCES}

Aramwit, P., Bang, N., Ratanavaraporn, J and Ekgasit, S. (2014). Green synthesis of silk sericin-capped silver nanoparticles and their potent anti-bacterial activity. Nanoscale research letters 9: 79-85. doi: 10.1186/1556-276X-9-79

Ariga, K., Li, M., Richards, G and Hill, J. (2011). Nanoarchitectonics: A conceptual paradigm for design and synthesis of dimension-controlled functional nanomaterials. Journal of Nanoscience and Nanotechnology 11: 1-13. doi: 10.1166/jnn.2011.3839

Baláž, M., Balážová, L., Daneu, N., Dutková, E., Balážová, M., Bujňáková, Z and Shpotyuk, Y. (2017). Plant-mediated synthesis of silver nanoparticles and their stabilization by wet stirred media milling. Nanoscale Research Letters 12:1-9. http://sci-hub.tw/10.1186/s11671-017-1860-z

Chirchir, K.D., Cheplogoi, K.P., Omolo, O.J and Langat, K.M. (2018). Chemical constituents of Solanum mauense (Solanaceae) and Dovyalis abyssinica (Salicaceae). International Journal of Biological and Chemical Sciences 12: 999-1007. doi: $10.4314 /$ ijbcs.v12i2.29 
Choi, Y., Kang, S., Cha, S.-H., Kim, H.-S., Song, K., Lee, Y.J., Kim, K., Kim, Y.S., Cho, S and Park, Y. (2018). Platycodon saponins from Platycodi Radix (Platycodon grandiflorum) for the green synthesis of gold and silver nanoparticles. Nanoscale Research Letters 13: 1-10. doi: 10.1186/s11671-018-2436-2

Chowdhury, S., Basu, A and Kundu, S. (2014). Green synthesis of protein capped silver nanoparticles from phytopathogenic fungus Macrophomina phaseolina (Tassi) Goid with antimicrobial properties against multidrug-resistant bacteria. Nanoscale Research Letters 9: 1-11. doi: 10.1186/1556-276X-9-365

Chung, I.-M., Park, I., Seung-Hyun, K., Thiruvengadam, M and Rajakumar, G. (2016). Plant-mediated synthesis of silver nanoparticles: their characteristic properties and therapeutic applications. Nanoscale Research Letters 11: 40-53. doi: org/10.1186/s11671-016-1257-4

Karsheva, M., Kirova, E and Alexandrova, S. (2013). Natural antioxidants from citrus mandarin peels. Extraction of polyphenols; effect of operational conditions on total polyphenols contents and antioxidant activity. Journal of Chemical Technology and Metallurgy 48: 35-41.

Kaviya, S., Santhanalakshmi, J., Viswanathan, B., Muthumary, J and Srinivasan, K. (2011). Biosynthesis of silver nanoparticles using Citrus sinensis peel extract and its antibacterial activity. Spectrochimica Acta Part A: Molecular and Biomolecular Spectroscopy 79: 594-598. doi: 10.1016/j.saa.2011.03.040

Kipngeno, C.D., Mshimba, S.M and Gilbert, C. (2014). Antimicrobial activity and phytochemical investigation of crude extracts of the fruits of Solanum incanum (Solananceae) and Dovyalis abbysinica (Flacourtiaceae). Science Journal of Microbiology 2014. doi: 10.7237/sjmb/193

Kumar, K.M., Mandal, B.K and Tammina, S.K. (2013). Green synthesis of nano platinum using naturally occurring polyphenols. RSC Advances 3: 4033-4039. https://doi.org/10.1039/C3RA22959A

Markus, J., Wang, D., Kim, Y.-J., Ahn, S., Mathiyalagan, R., Wang, C and Yang, D.C. (2017). Biosynthesis, characterization, and bioactivities evaluation of silver and gold nanoparticles mediated by the roots of Chinese herbal Angelica pubescens Maxim. Nanoscale Research Letters 12(1): 46-57. doi: 10.1186/s11671-017-1833-2

Masurkar, S.A., Chaudhari, P.R., Shidore, V.B and Kamble, S.P. (2011). Rapid biosynthesis of silver nanoparticles using Cymbopogan citratus (lemongrass) and its antimicrobial activity. Nano-Micro Letter 3: 189-194. http://scihub.tw/10.1007/BF03353671

Mohamed, R.M., Mckinney, D., Kadi, M.W., Mkhalid, I.A and Sigmund, W. (2016). Platinum/zinc oxide nanoparticles: enhanced photocatalysts degrade malachite green dye under visible light conditions. Ceramics International 42: 9375-9381. doi 10.1016/j.ceramint.2016.02.147

Nadagouda, M.N and Varma, R.S. (2008). Green synthesis of silver and palladium nanoparticles at room temperature using coffee and tea extract. Green Chemistry 10: 859-862. doi: 10.1039/B804703K

Rashid, M.U., Bhuiyan, M.K.H and Quayum, M.E. (2013). Synthesis of silver nano particles (Ag-NPs) and their uses for quantitative analysis of vitamin C tablets. Dhaka University Journal of Pharmaceutical Sciences 12: 29-33. doi: https://doi.org/10.3329/dujps.v12i1.16297 
Rodríguez-León, E., Iñiguez-Palomares, R., Navarro, R.E., Herrera-Urbina, R., Tánori, J., Iñiguez-Palomares, C and Maldonado, A. (2013). Synthesis of silver nanoparticles using reducing agents obtained from natural sources (Rumex hymenosepalus extracts). Nanoscale Research Letters 8: 318-325. doi: 10.1186/1556-276X-8-318

Safdar, M.N., Kausar, T., Jabbar, S., Mumtaz, A., Ahad, K and Saddozai, A.A. (2017). Extraction and quantification of polyphenols from kinnow (Citrus reticulate L.) peel using ultrasound and maceration techniques. Journal of Food and Drug Analysis 25: 488-500. doi: 10.1016/j.jfda.2016.07.010

Shija, K.M., Nondo, R.S., Mloka, D., Sangeda, R.Z and Bwire, G.M. (2020). Effects of lemon decoction on malaria parasite clearance and selected hematological parameters in Plasmodium berghei ANKA infected mice. BMC Complementary Medicine and Therapies 20(1): 24. doi: 10.1186/s12906-020-2820-1.

Siddiqi, K.S and Husen, A. (2016). Green synthesis, characterization and uses of palladium/platinum nanoparticles. Nanoscale Research Letters 11: 482. doi: 10.1186/s1 1671-016-1695-z

Songping, W and Shuyuan, M. (2005). Preparation of ultrafine silver powder using ascorbic acid as reducing agent and its application in MLCI. Materials Chemistry and Physics 89: 423-427. doi: 10.1016/j.matchemphys.2004.09.026.

Soundarrajan, C., Sankari, A., Dhandapani, P., Maruthamuthu, S., Ravichandran, S., Sozhan, G and Palaniswamy, N. (2012). Rapid biological synthesis of platinum nanoparticles using Ocimum sanctum for water electrolysis applications. Bioprocess and Biosystems Engineering 35: 827-833. doi: 10.1007/s00449-011-0666-0. 